\title{
Anti-D Prophylaxis Reviewed in the Erea of Foetal RHD Genotyping
}

\author{
Minon $\mathrm{JM}^{1^{*}}$, Gerard $\mathrm{CH}^{2}$, Chantraine $\mathrm{F}^{3}$ and Nisolle $\mathrm{M}^{3}$ \\ ${ }^{1}$ Department of Laboratory Medicine, Immunohematology laboratory and Blood Bank, CHR de la Citadelle, Bd du 12eme de Ligne, 1, Belgium \\ ${ }^{2}$ Department of Laboratory Medicine, Immunohematology Laboratory and Blood Bank, CHU de Liege, Sart-Tilman B35, 4000 Liege, Belgium \\ ${ }^{3}$ Department of Obstetrics and Gynecology, Fetal medicine unit, Université de Liège, CHR de la Citadelle, Bd du 12ème de Ligne, 1, 4000 Liege, Belgium \\ "Corresponding author: Jean-Marc Andre Minon, MD, PhD, Department of Laboratory Medicine, Immunohematology laboratory and Blood Bank, CHR de la Citadelle \\ Bd du 12eme de Ligne, 1, Belgium, Tel: 00324223 8781; E-mail: jean.marc.minon@chrcitadelle.be
}

Received date: Aug 05, 2015, Accepted date: Aug 31, 2015, Publication date: Sep 04, 2015

Copyright: (c) 2015 Minon JM, et al. This is an open-access article distributed under the terms of the Creative Commons Attribution License, which permits unrestricted use, distribution, and reproduction in any medium, provided the original author and source are credited.

\begin{abstract}
A few years ago, the prevention of anti-D immunization was currently based on systematic postnatal prophylaxis associated with targeted antenatal injection in high-risk situations of foeto-maternal haemorrhage. The failures of prevention are mainly due to the non-respect of established guidelines for RhIG prophylaxis, and to spontaneous undetected foetal-maternal haemorrhages without any obvious cause during the third trimester of pregnancy.

In order to reduce the rate of residual post-pregnancy anti-D immunization, several countries decided to associate the classical prophylaxis to a routine antenatal anti-D prophylaxis (RAADP) during the 28th or 29th week of gestation. Since about ten years, the foetal RHD genotyping in maternal plasma enables us to limit the antenatal prophylaxis only to those $D$ - women carrying a $D+$ foetus.

This paper deals with: the advantages of an antenatal prevention in the light of non invasive foetal RHD genotyping, the rules rendering prevention protocols efficient whatever the algorithm applied, and the recommended immuno-haematology follow-up of women who have received RhIG.
\end{abstract}

Keywords: Anti-D prophylaxis; Foetal RHD genotype; Haemolytic Disease of the foetus and Newborn

\section{Introduction}

The pathogenesis of haemolytic disease of the foetus and newborn (HDFN) was first elucidated by Levine (1941) thanks to the discovery of the Rh (Rhesus) blood group by Landsteiner and Wiener in 1940 $[1,2]$. He demonstrated the possibility of maternal immunization through placenta against foetal antigen(s) inherited from the father and lacking in the mother; in this particular case, the D antigen.

In 1940, prenatal mortality due to HDFN was around 4 out of 100 births, representing 10 percents of the global childhood mortality. Half of the affected foetuses died from hydrops foetalis or nuclear icterus. The improvement of the management of this pathology by experts reduced the mortality rate to less than 5 percent. The immunoprophylaxis of anti-D immunisation by injection of anti-D immunoglobulin's at delivery was only introduced in the late sixties [3].

Despite the application of this immuno-prophylaxis to $\mathrm{RhD}$ negative patients, HDFN due to anti-D still remains today the most frequent and severe in Europe [4].

In the absence of anti-D injection, a $\mathrm{D}$ negative mother bearing an $\mathrm{ABO}$ compatible $\mathrm{D}$ positive foetus has a $16 \%$ possibility of developing alloimmune anti-D. When the mother and the foetus are $\mathrm{ABO}$ incompatible (20\% of pregnancies), this rate falls to $2 \%$. So, globally, the risk for a D-negative woman to become immunized by a $\mathrm{D}$ positive foetus stands at around $13.2 \%$ [3].
However, when appropriate doses of anti-D immunoglobulin's are injected within 72 hours after delivery, the rate of immunisation is reduced by $90 \%$ and the residual risk is around 1 to $2 \%$ [5].

Most failures are due on one hand, to undetected foeto-maternal haemorrhages $(\mathrm{FMH})$ that occurs during the third trimester of pregnancy, and, on the other hand, (in more than $30 \%$ of cases), to disregard for prophylaxis rules [6].

When D negative patients are systematically injected at 28 weeks of pregnancy, the residual risk is further reduced by more than $60 \%$ [7].

Throughout pregnancy, foeto-maternal haemorrhage increases in frequency (from 3\% during the first trimester to $45 \%$ during the third one), and in volume $(<0.1 \mathrm{~mL}$ during the first trimester and in variable amounts during the third one) [8]. A dose-dependant correlation is known to exist between the volume of foeto-maternal haemorrhage and the occurrence of allo-immunisation. This is the reason why an adequate and timely administration of anti-D immunoprophylaxis remains essential. These practical aspects, including guidance regarding to routine antenatal anti-D prophylaxis (RAADP), are reviewed in this paper.

\section{Systematic Antenatal Prophylaxis}

\section{Cost/efficiency}

Prevention of anti-D allo-immunisation can be applied following at least two different approaches: the first one, as a postnatal prophylaxis after delivery of a $\mathrm{Rh} \mathrm{D}$ positive baby, in addition with targeted prophylaxis following potentially sensitizing events during pregnancy, 
and the second one, as a systematic antenatal prophylaxis at 28 weeks of gestation.

This matter has been discussed in a review published by Parant, who concluded that in the group without systematic antenatal prophylaxis, the global rate of immunisation turned around 1.5\% (1.2 to $1.9 \%)$, whereas in the group of patients who benefited from a systematic antenatal prophylaxis, the average rate of alloimmunization was around $0.3 \%$ (0 to $0.9 \%)$ [9]. These data are in favour of sytematic prevention at 28-29 weeks of gestation [10].

From an economical point of view, Canadian and English studies tend to show that systematic prevention involves greater drug and administrative costs than the targeted ones, but the cost/efficiency ratio still remains favourable [11]. The benefits of RAADP to be considered include: avoidance of anti-D sensitisation, reduction of incidence of haemolytic diseases, reduction of foetal/neonatal losses, avoidance of disability of the child, and positive effects of these outcome measures on the quality of life of the mother [12]. Most western countries recommend a systematic antenatal prophylaxis for all Rh D negative patients. However, so far, none of these studies ever organized an antenatal prevention which would be dedicated solely to those women bearing Rh D+ foetus. This eventuality is now rendered possible owing to the determination of foetal RHD from maternal plasma. It allows us to reduce, by around $40 \%$, the cost of unnecessary RhIG prenatal injections. This genotyping approach may offer additional cost benefits by reducing the immuno-haematological and sonographic follow-up of pregnancy [13]. The cost-effectiveness calculations of this approach will depend on the cost of foetal genotyping in maternal plasma. At present, this test is not refunded by the Health Service in Belgium except in anti-D alloimmunized mothers.

\section{Search for RHD foetal gene from maternal plasma}

Since 2002 in our hospital, the determination of foetal RHD from maternal plasma has been included in the biological follow-up of Rh D negative patient as early as the 12th week of gestation. We have reported $100 \%$ diagnostic accuracy in our non invasive foetal RHD genoyping assay by targeting multiple exons with real-time PCR [14,15]. The overall diagnostic accuracy was 96.5\% (95\% CI: 95.6-97.2) in the meta-analysis of Geifman-Holtzman, several studies also showing $100 \%$ diagnostic accuracy [16].

In order to avoid reporting a false negative result, the Ychromosome linked SRY gene sequence is amplified to confirm that foetal DNA is present in maternal plasma. A positive PCR signal ensures that the foetal DNA is present. This SRY-based internal control is only applicable for pregnancies carrying a male foetus. So, when the search for foetal RHD is negative and SRY gene absent, it is recommended to confirm the first result by using either a new blood sample a few weeks later or amniocytes when an amniotic liquid puncture has been programmed for another reason. This careful approach is justified by the absence of internal control for the Rh D negative female foetuses. It does not significantly differ from the basic rules in the matter of $\mathrm{ABO} / \mathrm{D}$ blood groups that require two different determinations before being validated.

The knowledge of foetal RHD status allows us to save up the injection of anti-D immunoglobulins at 28-29 weeks as well as those applied in the situations at risk of FMH among the $40 \%$ of $\mathrm{Rh} \mathrm{D}$ negative women bearing an RHD negative foetus.
On the other hand, genotyping of the foetal D gene also exempts D negative patients, bearing a $\mathrm{D}$ - foetus, to be exposed to any risk that might be associated with the administration of a human plasmaderived product. Moreover, there is worldwide shortage of anti-D [17].

As far as the post-partum immunoprophylaxis is concerned, determining the Rh D phenotype of a newborn from a $\mathrm{D}$ negative woman must continue to be determined even when the RHD foetal genotype has been searched during gestation [18].

Because samples collected in operating rooms are frequently mislabelled (newborn/mother inversion, cord blood contaminated by maternal blood, requisition mismatches), the French Society of Blood Transfusion (as well as the Society of Perinatal Medicine in France) recommends confirming the $\mathrm{D}$ negative phenotype of the baby determined on cord blood, by a second one performed on peripheral blood [19]. This should be strongly recommended when there was no previous RHD genotyping of the foetus during the pregnancy.

\section{Rules of Good Practice}

\section{Indications}

D negative women: Prophylaxis concerns all non immunized D negative women during and at the end of gestation. It has therefore no effect and is perfectly useless on previously immunized women. In rare cases of very weak anti-D which are solely detected by ultra sensitive techniques (enzymes) but remain undetectable by the anti-human globulin assay (AHG) in gel phase, the relevancy of prophylaxis must be discussed for each case individually. Usually, the follow-up in time allows us to decide the best approach.

Weak D women (previously named $\mathrm{D}^{\mathrm{u}}$ ): The term weak $\mathrm{D}(\mathrm{Du})$ is used to design a weakened expression of a "complete" D antigen (Figure 1). The molecular characteristic of the weak D types is single missense mutations which produce amino-acid exchange in the intracellular or transmembrane region of the D antigen [20]. The molecular techniques to predict foetal D status using maternal plasma, don't detect maternal weak D types and the maternal RHD gene appears "intact" as in D+ patients. The lower immunoreactivity of D antigen requires the indirect antiglobulin technique (IAT) for detection. However, at present time, most weak D are now detected by direct agglutination using monoclonal IgM anti-D reagents, or in the gel matrix technique and are considered as D positive [21]. The majority $(90 \%)$ of caucasian individuals presenting a weak D phenotype are weak types 1, 2, 3. Among European persons, these weak D types are always associated with the DCe or the DcE haplotypes. They cannot so far be anti-D immunized. Therefore these patients have to be treated as $\mathrm{D}$ positive, i.e.: transfused with $\mathrm{D}$ positive red cells and, following delivery of a D positive baby, not given anti-D immunoglobulin $[22,23]$.

Partial D women: The term partial D is used to refer to an incomplete $\mathrm{D}$ antigen in which some extracellular epitopes are missing (Figure 1). Partial D phenotypes are the results of gene rearrangements and/or senseless mutations in regions of RHD encoding portions of $\mathrm{D}$ protein external to the red cell membrane [20]. Individuals with a partial D phenotype can produce anti-D when they are exposed to $\mathrm{D}$ positive red blood cells (RBC). Therefore, it is advisable that they should be typed as D negative if they are candidates for transfusion or anti-D prophylaxis. Such a strategy is applied in our laboratories to type partial DVI, the most frequent D partial phenotype amongst white people. DVI presents also a weak expression of D and their 
carriers may be readily anti-D immunized [24]. In practice, most other partial $\mathrm{D}$ are usually classified as $\mathrm{D}$ positive in direct agglutination tests with currently available reagents and can often only be identified as having partial D antigen after and because they have produced antiD.

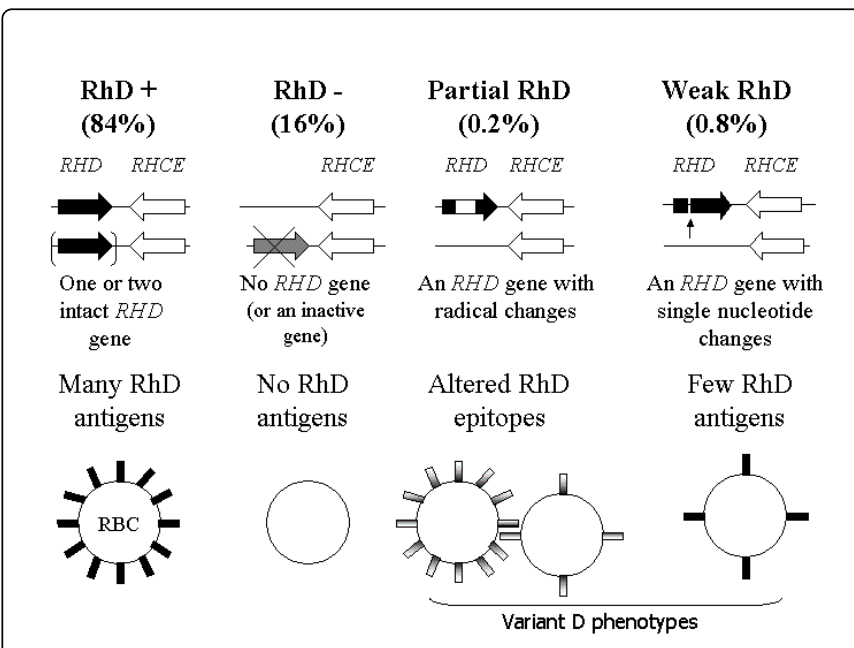

Figure 1: The genetic background for different $\mathrm{RhD}$ types (simplified). D negative Caucasians most often have a complete RHD gene deletion, but Africans and Asians have often an inactive RHD. The partial D red blood cells (RBC) demonstrate a normal number of Rh D antigens but with altered D epitopes. Many partial D results from hybrid genes with portions of RHD replaced by the corresponding portions of RHCE. The weak D red blood cells have a reduced number of complete $\mathrm{D}$ antigens. Weak $\mathrm{D}$ types primarily results from single point mutations in RHD. Some partial D (the most common in Caucasians: DVI) show both weak D and partial $\mathrm{D}$ features.

Pregnant women with suspected partial D or Weak D and molecular foetal RHD typing: Because some weak D expression reflects the presence of a partial $\mathrm{D}$ and because some carriers of rare weak D types may become immunized, the guidelines for prenatal and perinatal immunohaematology (edited by AABB) say that the weak D test by IAT should not be used to D type maternal samples [25]. Therefore, these patients with a weak expression of $\mathrm{D}$ are classified as D- and receive D- transfusion and RhIG prophylaxis. So, obstetricians send us some apparent D- women for foetal RHD genotyping. But molecular foetal RHD typing with maternal plasma should uncover a maternal "intact" RHD gene in some of these apparently D- women and the risk of erroneously defining the RHD gene as being of foetal origin should be considered [15]. Discrepancies between maternal Dphenotype and a maternal RHD+ genotype can create confusion in obstetricians' mind. On the other hand, our molecular techniques using maternal plasma detect the most of partial $\mathrm{D}$ with radical changes of the RHD gene. For these reasons, we now systematically test for weak D by IAT all D- women who are referred for foetal RHD genotyping and perform, from a maternal buffycoat DNA, a RHD genotyping of serologically D-, C+, and/or E+ European pregnant women. Those molecular tests distinguish common partial and weak $\mathrm{D}$ types and enable us not to give RhIG prophylaxis to pregnant women carrying prevalent weak D types 1,2 and 3. The partial D and other no types 1 to 3 weak D patients would be immunized when exposed to D+ blood. In this case, injection of anti-D immunoglobulin in pregnant women is proposed althought its efficiency in preventing sensitization has not been demonstrated.

Among European populations, about one percent of people carry variant RHD alleles producing weak D including some partial D $[26,27]$. In Black individuals, most $D$ variants with a weak expression are partial D and are often associated with Dce haplotype [28].

Weak D baby: All serologically D negative babies should be tested for the presence of weak D expression with the indirect antiglobulin technique. If the baby is weak $\mathrm{D}$, prophylaxis must be applied to $\mathrm{D}$ negative women, whereas it's not necessary if the baby is proven to be D negative.

\section{Detection of foetal erythrocytes}

The principle of the Kleihauer-Betke assay - the most widely used in Belgium - lies on the greater resistance to acid elution of foetal hemoglobin $(\mathrm{HbF})$ compared to adult hemoglobin ( $\mathrm{HbA})$. In practice, $\mathrm{HbA}$ is eluted from RBCs fixed on blood films, leaving red cell ghosts, so that the red cells containing $\mathrm{HbF}$ can be stained.

The volume of foetal RBC is calculated according to the following formula [29]:

\section{Volume of foetal $\mathrm{RBC}=2400 \times \mathrm{F} / \mathrm{A} \mathrm{mL}$,}

where $\mathrm{F} / \mathrm{A}$ is the proportion of foetal (F) RBC among maternal adult (A) RBC.

$$
\begin{aligned}
& -2400=1800 \times 1.22 \times 1.09 \mathrm{~mL} \\
& -1800 \mathrm{~mL}=\text { volume of maternal red blood cells, } \\
& -1.22=\text { foetal } \mathrm{RBC} \text { are } 22 \% \text { larger than adult } \mathrm{RBC}, \\
& -1.09=92 \%=\text { proportion of foetal } \mathrm{RBC} \text { stain darkly. }
\end{aligned}
$$

The volume of foetal blood (assuming a haematocrit of 50\%) can be deduced by multiplying the volume of foetal RBC by two.

Clinicians should be aware that the results of a Kleihauer-Betke test can be expressed on laboratory protocols either in terms of $\mathrm{mL}$ of fœtal RBC or in $\mathrm{mL}$ of fœtal whole blood. This is particularly important when the dose of anti-D immunoglobulins has to be adjusted according to the volume of the FMH. The general principe is that $100 \mu \mathrm{g}$ of RhIG is capable of suppressing sensitization by $4-5 \mathrm{ml}$ of foetal $\mathrm{RhD}$ positive red blood cells or by $8-10 \mathrm{ml}$ of foetal $\mathrm{RhD}$ positive whole blood.

The Kleihauer test' sensitivity allows to detect $7.2 \mathrm{~mL}$ of foetal $\mathrm{RBC}$ in the maternal circulation i.e. around $0.3 \%$ of fotal RBC, with a variation coefficient of around $10 \%$.

Some hemoglobinopathies (thalassemia, drepanocytosis, and hereditary persistence of foetal hemoglobin...) present varying resistance to acid elution and may entail false positive results. When such a case is suspected, some laboratories detect D positive foetal RBC by flow cytometry, by using fluorochrome coupled anti-D reagents. Flow cytometry presents a very high sensitivity $(0.01 \%$ i.e. $0.2 \mathrm{~mL}$ of foetal RBC), but this method is expensive, time consuming and not suitable for emergency cases.

At delivery or in situations at risk of FMH, prophylaxis must always be applied even when the result of the Kleihauer test is negative.

In practice, the search for foetal RBC should be performed within 2 hours of delivery (or a high-risk situation) but at least 30 minutes after 
placental evacuation to be sure that all the foetal erythrocytes have entered in maternal circulation [30].

Kleihauer's test results should always be transmitted to the clinician for those cases necessitating a supplementary dose, which should be administered within 72 hours post-delivery.

\section{Doses}

In Belgium, there is only one preparation of anti-D immunoglobulin (RhIgG) available (Rhogam): it contains $300 \mu \mathrm{g} / \mathrm{mL}$ of IM injectable RhIgG. $300 \mu \mathrm{g}$ is an amount of IgG capable of neutralizing 12 to $15 \mathrm{ml}$ of foetal RBC (i.e. 24 to $30 \mathrm{~mL}$ of foetal blood).

The routine antenatal anti-D prophylaxis consists of injecting 300 $\mu \mathrm{g}$ of anti-D at between 28 and 29 weeks of gestation.

After invasive examinations or accidents occurring before the 20th week, one IM dose is sufficient since the blood mass of a 20 weeks old foetus is lower than the volume neutralized by $300 \mu \mathrm{g}$.

After 20 weeks, there is a need to assess the volume of FMH and the dosage should be adapted according to the volume of circulating foetal RBC: $10 \mu \mathrm{g}$ per $0.5 \mathrm{~mL}$ foetal RBC or $1 \mathrm{~mL}$ foetal blood.

When the risk of FMH is low, for example after extra-placenta amniocentesis, it is not necessary to perform a Kleihauer test, and injection of one standard dose of anti-D is sufficient.

It is recommended to search for irregular antibodies (RAI) before the first injection of anti-D prophylaxis, even if the injection can be made before the RAI result is obtained. This allows for the detection of any anti-D allo-immunisation (and other than anti-D) which would have been undetectable earlier $[31,32]$.

\section{Delay for the injection of RhIgG}

To reach optimal efficiency, the injection of RhIG should be made as soon as possible within 72 hours following delivery or HFM. If it has been omitted, injection of RhIgG can still be performed until 28 days after delivery, but the later it is done, the less efficient it will be [33].

\section{Control of RhIG efficiency}

The sole relevant control test is the search for foetal RBC in maternal blood 48 hours after injection of RhIG [10]. RhIG has been efficient when all foetal RBC have disappeared. This control is only necessary when a supplementary dose has to be injected i.e. when the foetal hemorrhage was greater than $15 \mathrm{~mL}$ of foetal RBC.

There are no arguments at all to say that the presence of passive anti-D in maternal circulation can be considered as reflecting the efficiency of RhIG injection; it only reflects the fact that the injection has really been made.

Within three to seven days after IM injection of RhIG, the titre of anti-D in maternal blood can reach $1 / 4$ (when measured by using the reference method i.e. IAT in saline). Antibody titration decreases thereafter as a function of both the half-life time of IgG (21 days), and the rate of consumption by D positive foetal RBC. However, a residual activity of RhIG can be demonstrated in some maternal plasma during several months.
If any doubt remains concerning the appropriate dosage of RhIgG administered, the clinician can register the situation in the light of both the results of the Kleihauer test and the presence of anti-D in maternal blood (Table 1). Absence of detectable anti-D has contrarily to its presence-a predictive value in terms of absence of protection.

\begin{tabular}{|l|l|l|}
\hline $\begin{array}{l}\text { Foetal red blood } \\
\text { cells (Kleihauer) }\end{array}$ & $\begin{array}{l}\text { Free circulating anti- } \\
\mathbf{D}^{*} \text { in maternal blood }\end{array}$ & Action \\
\hline 0 & No & $2^{\text {nd }}$ Injection \\
\hline 0 & Yes & No $2^{\text {nd }}$ injection \\
\hline Yes & No & $\begin{array}{l}\text { Assess FMH } \\
\text { Inject appropriate dose }\end{array}$ \\
\hline Yes & Yes & assess FMH at $48 \mathrm{~h}$ \\
\hline *IAT tube method in phosphate buffered saline \\
\hline
\end{tabular}

Table 1: After an initial injection of RhIG, when a second one should be injected?

One should also keep in mind that several factors can affect the clearance of fetal $\mathrm{RBC}$, including: a laboratory failure at the time of the initial foetal RBC count, a dizygotic twin pregnancy with one D positive and one $\mathrm{D}$ negative foetus, a weak $\mathrm{D}$ expression or a splenectomized woman [34].

Finally, so many different situations exist - which can entail error, omission or default - that it is more reasonable to promote a systematic injection of RhIgG at delivery even when the patient has already received one before.

\section{Immuno-Haematologic Features of RhIG Injections}

\section{In maternal blood}

Residual anti-D: Sometimes, a weak anti-D activity may be detected in maternal serum for several weeks by IAT and, by more sensitive techniques, for several months after RhIgG injection, and persistent passive anti-D may mask an early active allo-immunisation. On the other hand, the clearance of passive anti-D is highly variable from one patient to another. Therefore, a low titration of anti-D $(<1 / 4$ by IAT) does not allow to discriminate passive and active immunisation [25].

To provide a basis for distinguishing between prophylactic and immune anti-D, all D negative pregnant women should benefit before the RAADP, at 28 weeks gestation, from a screening for red cell alloantibodies. Moreover, it is essential for the biologist to be informed to any previous administration of RhIgG in the current pregnancy, including date and dose, at the time of analysis, in order to interpret the anti-D titre. It is generally admitted that anti-D titration higher than $1 / 4$ is highly predictive of an active immunisation. When titration results are not conclusive, it is necessary to repeat the analysis a few weeks later to be able to conclude to passive or active immunisation. Prophylactic anti-D levels will fall with time whereas immune anti-D levels will remain stable or rise. Some authors propose to perform micro titration to differentiate an active from a passive anti-D in the follow-up of RhIG prophylaxis [35].

In practice, any laboratory request form for the search for irregular antibodies during pregnancy should indicate if the patient has or not 
received RhIG, and if so, when. Besides, injection of RhIG should systematically be noted in the medical history of the patient even though this is not (yet) mandatory in Belgium.

Calendar for the research of irregular antibodies during pregnancy: In the absence of any sensitizing event or clinical indication, there is no need to repeat an antibody screening at the 8th or 9th month when a systematic prophylaxis, preceded by a screening for red cell alloantibodies, has been applied at 28 weeks [36].

However, the introduction of RAADP has resulted in a positive antibody screen with the detection of anti-D in samples taken after 28 weeks gestation. A panel of D negative cells should be used to exclude the presence of unexpected alloantibodies of other specificities. If the mother needs blood transfusion in a context of obstetrical haemorrhage in the delivery, this would lengthen the delay before blood product can be delivered. Therefore to ensure an appropriate blood management of obstetrical haemorrhage, which are often sudden and unexpected, a search for irregular antibodies can be suggested within the 7 days preceding delivery in order to identify alloantibodies other than pass if anti-D possibly present in the serum of the mother $[37,38]$. In presence of clinically relevant alloantibodies, a compatibility testing must be performed [39].

\section{Hindrances}

Plasma used to prepare anti-D immunoglobulins is of human origin; they are collected from immunized blood donors and therefore may contain Rhesus antibodies other than anti-D: anti-C or anti-E, for example. That is the reason why it may occur that anti-C or anti-E be detected in the serum of injected women [34].

\section{In the newborn}

Anti-D prenatal prophylaxis has no effect on the foetus. At most, foetal red blood cells can be sensitized, which will lead to a positive direct Coombs test at birth, but without any clinical consequences [40]. However, if multiple doses are given, it is recommended that bilirubinemia should be followed.

\section{Conclusion}

The possibility to know the RHD foetal genotype from a maternal blood sample during pregnancy should dramatically modify the recommendations in the matter of prophylaxis for foetal-maternal allo-immunization.

Associated with the routine antenatal anti-D prophylaxis at 28-29th week of gestation, it is possible now to propose a new approach of $\mathrm{D}$ negative pregnant women (Figure 2).

RHD foetal genotyping during pregnancy allows us to recommend a prevention policy targeted at the $61 \%$ of $\mathrm{D}$ - women bearing a $\mathrm{D}+$ foetus, by systematically injecting anti-D immunoglobulin during the third trimester of pregnancy. At the same time, knowing the RHD foetal status allows to avoid injections in $39 \%$ of women carrying a Dfoetus.

It is highly regrettable to see that prevention failures are mainly due to human or functional negligence: default of injection, too late injection, and so on.

At the time of accreditation, it was probably worth reminding ourselves of the best practices for a high-risk pregnancy follow-up: the predictive value of antibody screening, the significance of circulating specific antibodies, the results of Kleihauer, which allows us to measure the range of foetal-maternal haemorrhage and to adjust the dose of RhIG to inject.

Even if any best practice rule will never replace the medical judgement of clinicians, it should be taken into consideration how they have progressed and benefited from technological advances such as the foetal RHD genotyping obtained from a non invasive examination.

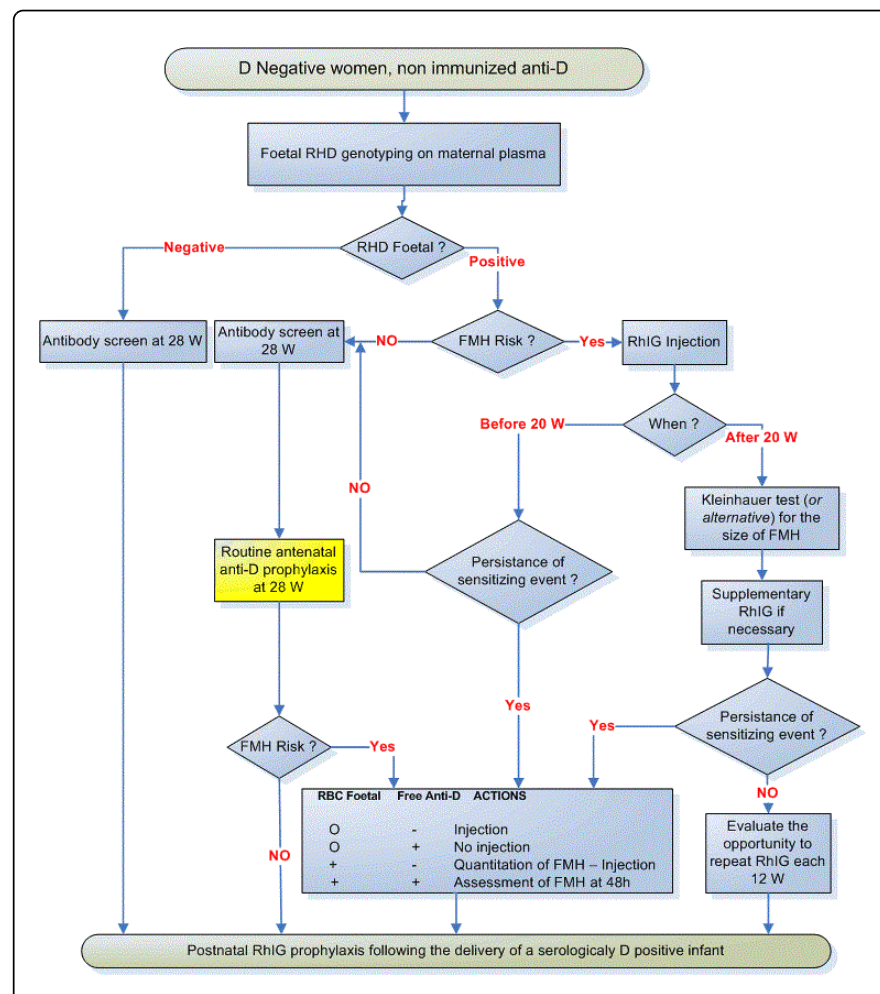

Figure 2: Algorithm for RhIG prophylaxis in pregnant D- women.

\section{References}

1. Levine P, Katzin E, Burnham L (1941) Isoimmunisation in pregnancy: its bearing on the aetiology of erythroblastosis fetalis. JAMA 116: 825-827.

2. Landsteiner K, Wiener A (1940) An agglutinate factor in human blood recognized by immune sera for rhesus blood. Proc Soc Exp Biol Med 43: 223.

3. Bowman J (2003) Thirty-five years of Rh prophylaxis. Transfusion 43: 1661-1666.

4. Daniels G (2002) Blood group antibodies in haemolytic disease of the fetus and newborn. Alloimmune disorders of pregnancy. Cambridge: University Press: 21-40.

5. Ratsimbazagy V, Alba J, Cohen J (2002) L'allo-immunisation foetomaternelle anti-D. Gynecol Obstet 450: 20-23.

6. [No authors listed] (1999) ACOG practice bulletin. Prevention of Rh D alloimmunization. Number 4, May 1999 (replaces educational bulletin Number 147, October 1990). Clinical management guidelines for obstetrician-gynecologists. American College of Obstetrics and Gynecology. Int J Gynaecol Obstet 66: 63-70.

7. Chilcott J, Lloyd Jones M, Wight J, Forman K, Wray J, et al. (2003) A review of the clinical effectiveness and cost-effectiveness of routine anti$\mathrm{D}$ prophylaxis for pregnant women who are rhesus-negative. Health Technol Assess 7: iii-62. 
8. Bowman JM, Pollock JM, Penston LE (1986) Fetomaternal transplacental hemorrhage during pregnancy and after delivery. Vox Sang 51: 117-121.

9. Parant O (2006) Comparaison de l'efficacit des diffrentes formes de prvention de l'allo-immunisation anti-D au cours de la grossesse: prvention cible limite aux situations risque ou associe une prvention systmatique au 3e trimestre. J Gynecol Obstet Biol Reprod (Paris). 35: 1S93-91S103.

10. Cortey A, Brossard Y (2006) [Prevention of fetomaternal rhesus-D alloimmunization. Practical aspects]. J Gynecol Obstet Biol Reprod (Paris) 35: 1S123-121S130.

11. Ravinet J, Carbonne B (2006) [Economic analysis of the prevention of anti-D immunization]. J Gynecol Obstet Biol Reprod (Paris) 35: 1S104-101S111.

12. https://www.nice.org.uk/.

13. Carbonne B, Cortey A, Rouillac-Le Sciellour C, Brossard Y (2008) [Non invasive fetal $\mathrm{RhD}$ genotyping using maternal blood: time for use in all RhD negative pregnant women]. Gynecol Obstet Fertil 36: 200-203.

14. Minon JM, Schaaps JP, Retz MC, Dricot JF, Foidart JM, et al. (2005) [Prenatal determination of fetal RHD in maternal plasma: two-years experience of routine clinical use]. J Gynecol Obstet Biol Reprod (Paris) 34: 448-453.

15. Minon JM, Gerard C, Senterre JM, Schaaps JP, Foidart JM (2008) Routine fetal RHD genotyping with maternal plasma: a four-year experience in Belgium. Transfusion 48: 373-381.

16. Geifman-Holtzman O, Grotegut CA, Gaughan JP (2006) Diagnostic accuracy of noninvasive fetal $\mathrm{Rh}$ genotyping from maternal blood--a meta-analysis. Am J Obstet Gynecol 195: 1163-1173.

17. van der Schoot CE, Hahn S, Chitty LS (2008) Non-invasive prenatal diagnosis and determination of fetal $\mathrm{Rh}$ status. Semin Fetal Neonatal Med 13: 63-68.

18. Cortey A, Brossard Y, Beliard R, Bourel D (2006) [Prevention of fetomaternal rhesus-D allo-immunization. Perspectives]. J Gynecol Obstet Biol Reprod (Paris) 35: 1S119-111S122.

19. Mannessier L, Alie-Daram S, Roubinet F, Brossard Y; Groupes de travail Immunohématologie de la Société française de transfusion sanguine et de la Société française de médecine périnatale (2000) [Prevention of fetal hemolytic disease: it is time to take action]. Transfus Clin Biol 7: 527-532.

20. Wagner FF, Flegel WA (2004) Review: the molecular basis of the Rh blood group phenotypes. Immunohematology 20: 23-36.

21. Williams M (2000) Monoclonal reagents for rhesus-D typing of Irish patients and donors: a review. Br J Biomed Sci 57: 142-149.

22. Flegel WA, Wagner FF (2002) Molecular biology of partial D and weak D: implications for blood bank practice. Clin Lab 48: 53-59.

23. Flegel WA (2006) Molecular genetics of RH and its clinical application. Transfus Clin Biol 13: 4-12.

24. Wagner FF, Gassner C, Muller TH, Schonitzer D, Schunter F, et al (1998) Three molecular structures cause rhesus D category VI phenotypes with distinct immunohematologic features. Blood 91: 2157-2168.
25. https://www.karger.com/Book/Home/231653.

26. Wagner FF, Gassner C, Müller TH, Schönitzer D, Schunter F, et al. (1999) Molecular basis of weak D phenotypes. Blood 93: 385-393.

27. Wagner FF, Kasulke D, Kerowgan M, Flegel WA (1995) Frequencies of the blood groups ABO, Rhesus, D category VI, Kell, and of clinically relevant high-frequency antigens in south-western Germany. Infusionsther Transfusionsmed 22: 285-290.

28. Chen Q, Flegel WA (2005) Random survey for RHD alleles among D+ European persons. Transfusion 45: 1183-1191.

29. British Committee for Standards in Haematology (2000) Guidelines for blood grouping and red-cell antibody testing during pregnancy and for performing red-cell alloantibody titrations. Oxford: Blackwell Science Ltd: 201-206.

30. Judd WJ, Scientific Section Coordinating Committee of the AABB (2001) Practice guidelines for prenatal and perinatal immunohematology, revisited. Transfusion 41: 1445-1452.

31. Hartwell EA (1998) Use of Rh immune globulin: ASCP practice parameter. American Society of Clinical Pathologists. Am J Clin Pathol 110: 281-292.

32. Banks AA (2002) Hemolytic Disease of the Newborn Methods In: Banks AAoB, ed. Technical Manual. (14thedn) Bethesda 2002 :730-731.

33. Bowman JM (1985) Controversies in Rh prophylaxis. Who needs $\mathrm{Rh}$ immune globulin and when should it be given? Am J Obstet Gynecol 151: 289-294.

34. Brossard Y, Parnet-Mathieu F, Larsen M (2000) Incompatibility foetomaternelles arythrocytaires. John Libbey Eurotext: 294.

35. Dupont M, Gouvitsos J, Dettori I, Chiaroni J, Ferrera V (2007) [Microtitration of anti-RH1 antibodies: interest in the follow-up of pregnant women]. Transfus Clin Biol 14: 381-385.

36. British Committee for Standards in Haematology Blood Transfusion Task Force, Gooch A, Parker J, Wray J, Qureshi H (2007) Guideline for blood grouping and antibody testing in pregnancy. Transfus Med 17: 252-262.

37. Sherman SJ, Greenspoon JS, Nelson JM, Paul RH (1993) Obstetric hemorrhage and blood utilization. J Reprod Med 38: 929-934.

38. Chapman JF, Elliott C, Knowles SM, Milkins CE, Poole GD; Working Party of the British Committee for Standards in Haematology Blood Transfusion Task Force (2004) Guidelines for compatibility procedures in blood transfusion laboratories. Transfus Med 14: 59-73.

39. Mannessier L (2007) [Immunohematologic surveillance of the pregnant woman and the new prevention policy of anti-RH1 allo-immunization]. Transfus Clin Biol 14: 112-119.

40. Maayan-Metzger A, Schwartz T, Sulkes J, Merlob P (2001) Maternal anti$\mathrm{D}$ prophylaxis during pregnancy does not cause neonatal haemolysis. Arch Dis Child Fetal Neonatal Ed 84: F60-62.
This article was originally published in a special issue, entitled: "Rh Blood Group System", Edited by Smita Mahapatra, SCB Medical College \& Hospital, India 